\title{
Indicadores de salud y bienestar social en población inmigrante y chilena: un estudio comparativo
}

\author{
Héctor Arancibia; Manuel Cárdenas; William Durán; Paula Eguren
}

Cómo citar este artículo:

Arancibia, H., Cárdenas, M., Durán, W., \& Eguren, P. (2021). Indicadores de salud y bienestar social en población inmigrante y chilena: un estudio comparativo. Acta Colombiana de Psicología, 24(1), 72-85. https://www.doi.org/10.14718/ACP.2021.24.1.7

Recibido, febrero 13/2020; Concepto de evaluación, junio 11/2020; Aceptado, junio 22/2020

\author{
Héctor Arancibia \\ ORCID: https://orcid.org/0000-0003-2850-1424 \\ Universidad de Valparaíso, Valparaíso, Chile. \\ Manuel Cárdenas \\ ORCID: https://orcid.org/0000-0002-5484-0078 \\ Universidad de Talca, Talca, Chile. \\ William Durán \\ ORCID: https://orcid.org/0000-0002-0235-0093 \\ Pontificia Universidad Javeriana, Bogotá, Colombia. \\ Paula Eguren \\ ORCID: https://orcid.org/0000-0003-2711-7500 \\ Universidad Alberto Hurtado, Santiago, Chile.
}

\begin{abstract}
Resumen
En el presente estudio se comparan los niveles medios de salud y bienestar social de adultos nacidos en Chile $(n=155)$ e inmigrantes de nacionalidad colombiana $(n=166)$, peruana $(n=154)$ y venezolana $(n=180)$. Los resultados están en línea con la "hipótesis selectiva de la inmigración", pues se encontraron mayores niveles de salud $\left(M_{\text {inmigrantes }}=40.35\right.$; $\left.M_{\text {chilenos }}=38.93 ; t[203.579]=2.745, p<.01\right)$ y bienestar social en inmigrantes $(M=88.62)$ que en chilenos $(M=85.42$; $t(190.778)=-3.197, p<.01)$. En general, en este trabajo se muestra el efecto negativo que tiene la percepción de discriminación sobre la salud, y el efecto positivo de las variables socioculturales de identidad endogrupal, enriquecimiento cultural y cercanía intergrupal sobre el bienestar social en personas inmigrantes.

Palabras clave: bienestar social, salud, aculturación, inmigración latinoamericana, discriminación, contacto intergrupal.
\end{abstract}

\section{Health and social well-being in immigrants and Chileans: a comparative study}

\begin{abstract}
This study compares the average health and social welfare levels of adults born in Chile $(\mathrm{n}=155)$ and immigrants of Colombian $(n=166)$, Peruvian $(n=154)$, and Venezuelan $(n=180)$ nationality. The results are in line with the "selective hypothesis of immigration," since higher levels of health (Minmigrants $=40.35$; Mchilenos $=38.93 ; \mathrm{t}[203,579]=2,745$, $p<.01)$ and social welfare were found in immigrants $(\mathrm{M}=88.62)$ than in Chileans $(\mathrm{M}=85.42 ; \mathrm{t}(190,778)=-3,197$, $p<.01)$. In general, this work shows the negative effect that the perception of discrimination has on health, and the positive effect of the socio-cultural variables of endo-group identity, cultural enrichment, and inter-group closeness on the social welfare of immigrants.

Keywords: social well-being, health, acculturation, Latin American immigration, discrimination, inter-group contact.
\end{abstract}

1 C/Serrano 546, Valparaíso, Chile. Tel.: (+56)322995813. Correo electrónico: hector.arancibia@uv.cl Esta investigación se realizó como parte del proyecto FONDECYT n. ${ }^{\circ} 3170187$ financiado por la Comisión Nacional de Investigación Científica y Tecnológica (CONICYT), Chile. 


\section{Introducción}

Un factor común en el proceso migratorio es la vivencia de un quiebre en la trayectoria vital de la persona, cuyo efecto trasciende a su entorno más inmediato, como el de la familia y amigos. Este quiebre refiere, en particular, a un rompimiento con las relaciones cotidianas de afecto y reconocimiento mutuo, con las redes sociales de apoyo, con la propia cultura, y con todo lo que conlleva a un adecuado funcionamiento social (Berry, 2005). En definitiva, es un quiebre con la historia de esa persona en su comunidad y con la seguridad propia de conocer y reconocer los códigos distinguibles que se han adquirido y transmitido durante años de socialización. Desde este lugar comienza la experiencia de aculturación, en la cual la persona se hace parte de una serie de procesos cuyos impactos se observan a nivel individual - como en la autoestima - intragrupal — como en la identificación endogrupal o la autoestima colectiva (Crocker et al., 1994) e intergrupal — como el favoritismo endogrupal (Turner et al., 1979)_; y que suelen tener un impacto negativo en el bienestar y en la salud de las personas migrantes (Huynh \& Fuligni, 2010; Jasinskaja-Lahti et al., 2006; Sevillano et al., 2014; Williams \& Mohammed, 2009).

Por otra parte, si bien la mayor parte de las investigaciones dan cuenta de niveles más bajos de salud y de bienestar en población inmigrante, estos resultados no son concluyentes (Bobowik et al., 2015; Parker-Frisbie et al., 2001; Pascoe \& Smart-Richman, 2009). De hecho, se han encontrado mejores indicadores de salud en inmigrantes, lo cual, desde la perspectiva de Cho et al. (2004), se explica por la denominada "hipótesis selectiva de la inmigración", según la cual los inmigrantes presentan niveles de salud por sobre la media al momento de emigrar, lo que les permite afrontar de mejor manera el proceso migratorio. Al respecto, Franzini et al. (2001) explican que los mejores niveles de salud — de la población hispana en EE. UU., en este caso-, lo que se ha denominado como la "paradoja de salud hispánica", son resultado de una fuerte identidad endogrupal y de una estrecha red de apoyo social que se da en este grupo que sirven para paliar los efectos de las condiciones económicas desfavorables que viven en comparación con la de los estadounidenses de etnia blanca. Sumado a esto, y desde una perspectiva cultural, se ha dicho que los inmigrantes que menos adoptan los códigos culturales de la sociedad de acogida presentan mejores niveles de salud (Hennessy-Burt et al., 2011).

Indicadores de bienestar social y de salud mental y fisica

Se han utilizado diversas pruebas para valorar y comparar los niveles de bienestar y salud en población inmigrante.
Una ampliamente utilizada ha sido la Escala de salud $S F-12$ (Chiu et al., 2009; Hoffmann et al., 2005; Nesterko et al., 2019), pues permite obtener tanto una medida general de salud como una medida de salud física y mental.

A las medidas objetivas de salud tradicionalmente utilizadas se les han incorporado otras que apuntan a evaluar la presencia de elementos favorables del bienestar; una perspectiva que ha surgido con fuerza dentro del marco de la psicología positiva, y que rompe con el énfasis en la patología y en la cura, para dirigirlo hacia los recursos que la persona ha desarrollado y que la hacen florecer en contextos habituales (Seligman \& Csikszentmihalyi, 2000).

Una de estas mediciones es la evaluación del "bienestar social", en la cual se pone un énfasis especial en la interacción intersubjetiva entre el individuo y su contexto (Blanco \& Díaz, 2005), en la importancia de la estructura social en la cual está inserta la persona, así como en su funcionamiento social, y en la explicación sobre cómo la sociedad contribuye a su propio bienestar (Keyes, 2006).

En particular, según Keyes (1998), el bienestar social se define como la evaluación que hacen las personas de las circunstancias sociales y de su funcionamiento dentro de la sociedad, y está constituido por cinco dimensiones: (a) la integración social, que refiere al sentimiento de pertenencia y de ser aceptado; (b) la aceptación social, que se relaciona con la aceptación de la naturaleza humana y la confianza depositada en los otros; (c) la contribución social, que se entiende como una creencia en el potencial y en el desarrollo de la sociedad conjuntamente - con un sentimiento de esperanza sobre el futuro-; (d) la actualización social, que se vincula a la evaluación de la trayectoria y del potencial de la sociedad; y, finalmente, (e) la coherencia social, que se entiende como el conocimiento y entendimiento de la vida social.

\section{Inmigración y salud en el contexto chileno}

Históricamente, el porcentaje de inmigrantes en Chile ha sido bajo, ya que ha alcanzado un promedio de $1.6 \%$ entre los años 1920 y 2002, con su punto más bajo en el año 1982 —en plena dictadura cívico-militar - con el $0.7 \%$ (Instituto Nacional de Estadísticas [INE], 2019). Hasta la primera década del siglo XXI, las personas inmigrantes provenían principalmente de países fronterizos - como Perú, Bolivia y Argentina-, y a ellos se sumaban inmigrantes europeos —españoles, italianos, alemanes, británicos-, sirios, palestinos, libios, entre otros, que llegaron a Chile principalmente durante el siglo XIX y mediados del XX.

En el contexto de esta inmigración, llama la atención la ley decretada en el año 1953 por el presidente Ibáñez del Campo, cuyo objetivo era el de promover la inmigración 
europea para contribuir a "perfeccionar las condiciones biológicas de la raza" (Centros de Estudios de Opinión Ciudadana [CEOC], 2009, p. 7); un emblemático episodio legislativo que da cuenta de las diferencias que ha tenido - y que aún tiene - la inserción de personas inmigrantes en la sociedad chilena, dependiendo del origen y los rasgos fenotípicos diferenciados entre el blanco europeo y el indígena o afrodescendiente (Canales, 2019; Cárdenas, 2006).

No obstante, el panorama de la inmigración en Chile ha cambiado considerablemente. Actualmente, el porcentaje de inmigrantes se ha triplicado, del $2.3 \%$ en 2014 (Departamento de Extranjería y Migración [DEM], 2016) al $6.7 \%$ estimado para el año 2018 (DEM, 2019), y se ha concentrado en términos relativos en las regiones de Tarapacá (13\%) y Antofagasta (11\%), aun cuando el $65 \%$ de los migrantes reside en la región Metropolitana.

De igual forma, el patrón migratorio, que se había caracterizado por la prevalencia de la migración fronteriza, también ha cambiado sustancialmente, pues en la actualidad tres de los cuatro grupos de mayor presencia en Chile pertenecen a algún país no fronterizo, siendo estos Venezuela $\left(1 .^{\circ}\right)$, Haití $\left(3 .^{\circ}\right)$ y Colombia $\left(4 .^{\circ}\right)$, de lo cual destaca que el segundo lugar lo ocupan los peruanos, quienes históricamente habían liderado el número de inmigrantes en Chile (INE, 2018).

Por otra parte, en cuanto al origen étnico, durante los últimos años se ha producido un arribo considerable de inmigrantes de etnia afrodescendiente, principalmente provenientes de Haití, Colombia, Ecuador y República Dominicana; y el flujo migratorio de los últimos años se ha caracterizado por una alta concentración en la llegada de inmigrantes en un acotado tramo temporal, pues el $67 \%$ de la actual población migrante llegó a Chile entre los años 2010 y 2017, de los cuales el $61 \%$ lo hizo entre el 2015 y 2017 (INE, 2018).

Más allá de los cambios observados en los últimos años, lo que pareciera mantenerse estable son las difíciles y desfavorables condiciones que experimentan a diario los inmigrantes residentes en Chile, pues, (a) los indicadores de prejuicio y discriminación continúan estables a lo largo del tiempo (Arancibia et al., 2016; Cárdenas, 2006; Cárdenas et al., 2011; Márquez, 2014); (b) se observan condiciones laborales precarias caracterizadas por ausencia de contratos, incumplimiento en el pago de imposiciones en la seguridad social, e incumplimiento de las condiciones laborales de la legislación chilena (Ramírez-Santana et al., 2019; Stefoni, 2009); (c) aún existen importantes barreras para el acceso a los servicios de salud (Cabieses et al., 2017; Cabieses \& Oyarte, 2020) y se encuentra un trato discriminatorio importante hacia la población migratoria (Concha \& Mansilla, 2017); y (d), es común encontrar una permanente vinculación de la tríada inmigración-delincuencia-expulsión por parte de los medios de comunicación (Stang \& Stefoni, 2016; Valdes, 2018) y de las autoridades gubernamentales, estas últimas más preocupadas por la deportación de personas inmigrantes que por su integración (El Mostrador, 2019).

En el caso de inmigrantes afrodescendientes, la experiencia de discriminación y racismo se experimenta aún con más fuerza (Gissi et al., 2019; Segovia \& Lufin, 2013). Por el color de piel, y en algunos casos por no dominar el idioma - p. ej., los haitianos—, se les deshumaniza y se les atribuyen más atributos de carácter sexual y físico que de carácter intelectual (Rojas et al., 2015; Tijoux, 2014); dinámicas de prejuicio y racismo que se trasladan también a los entornos educativos (Pavez-Soto et al., 2020; Salas et al., 2017; Tijoux, 2013) y a los de formación del profesorado, donde se observan actitudes implícitas negativas hacia la educación multicultural (Navas \& Sánchez, 2010).

\section{Perfil sociodemográfico de la población inmigrante en Chile}

La tradicional inmigración fronteriza experimentada en Chile se ha ampliado a otros inmigrantes latinoamericanos. Según el INE (2018), las personas migrantes en promedio son más jóvenes que los chilenos - $3.2 \%$ de personas mayores de 65 años, en comparación con el $11.8 \%$ de la población nacional- pues se encuentran entre los 20 y 45 años, de manera que compensan los desequilibrios de la pirámide poblacional local. Asimismo, se observa una leve mayoría de mujeres $(50.6 \%$ ) y un promedio de escolaridad mayor que el de los nacionales — para los nacionales es de 11 años, mientras que para las personas inmigrantes es de 12.6 años - Si se analiza la información de manera segmentada, el porcentaje de nacionales con educación básica o primaria es del $26.1 \%$, mientras que este corresponde al $12.8 \%$ en la población inmigrante; $\mathrm{y}$, al analizar los datos censales, solo el $29.1 \%$ de los chilenos declaró tener estudios superiores, mientras que en las personas inmigrantes este porcentaje fue mucho más alto - el 42.6\%-(INE, 2018).

No obstante, en términos de su inserción laboral, las personas inmigrantes se instalan mayoritariamente en el sector terciario que contempla las empresas, instituciones 0 actividades por cuenta propia y que pertenecen al área de servicios de la economía (87.8 \%) - ; a la vez que se observa una alta presencia de hogares unipersonales (32.8\%), así como condiciones de vivienda que dan cuenta de un $20 \%$ de hacinamiento entre los hogares de inmigrantes, en comparación con el 7 \% de la población local.

\section{Salud e indicadores de bienestar en Chile}

A pesar de las desfavorables condiciones a las cuales deben enfrentarse las personas inmigrantes en Chile, los 
indicadores de salud y de bienestar no siempre se ven afectados. En algunos casos, la condición de inmigrante opera más bien como un factor protector que de riesgo.

Por ejemplo, en la investigación realizada por Rojas et al. (2011) con una muestra constituida principalmente por inmigrantes peruanos adultos, el $17.8 \%$ presentaba algún trastorno mental, cifra que está muy por debajo del $50 \%$ de la prevalencia observada en la población chilena (Vicente et al., 2007) —una de las más altas a nivel mundial. Asimismo, según Cabieses et al. (2017), en el caso de los partos de mujeres inmigrantes, a pesar del tardío acceso a los controles del embarazo y al mayor riesgo psicosocial, el $28 \%$ presenta algún problema, a diferencia del $44 \%$ observado en los partos de mujeres chilenas. En contraste, las cifras de niños inmigrantes de 1 a 6 años no son alentadoras, pues el $25 \%$ de las altas hospitalarias por traumatismo corresponde a niños inmigrantes, en comparación con el $13 \%$ de los chilenos, lo cual puede explicarse por la condición de pobreza multidimensional en la que viven (Cabieses et al., 2017).

En cuanto a los indicadores de salud mental y bienestar, buena parte de los estudios cuantitativos realizados en Chile comparan los indicadores entre población inmigrante, especialmente entre la peruana y colombiana y, en menor medida, la boliviana. De los escasos estudios encontrados, uno de ellos muestra resultados similares para los peruanos y colombianos en prácticamente todas las dimensiones (Urzúa, Boudon-Torrealba et al., 2017), y un segundo estudio da cuenta de mejores indicadores para la muestra de colombianos en salud física y mental (Urzúa, Caqueo-Urízar, et al., 2017). En ninguno de los estudios se observaron diferencias significativas por género.

Por otra parte, respecto a los indicadores de bienestar psicológico y su relación con las estrategias de aculturación, Mera-Lemp et al. (2020) realizaron un estudio en el que reportan que los mejores indicadores de bienestar, en todas sus dimensiones, son los que presentan las personas que utilizan la estrategia de aculturación basada en la integración - entendida como la igual valoración de los aspectos de la cultura propia como los de la sociedad de acogida - mientras que quienes desarrollan una estrategia de asimilación —entendida como una sobrevaloración de los aspectos culturales de la sociedad de acogida por sobre los propios - presentan los peores indicadores de bienestar psicológico (Urzúa, Boudon-Torrealba et al., 2017; Yañez \& Cárdenas, 2010). En esta misma línea, la estrategia de consumo cultural - vinculada con una estrategia asimiladora - también estaría relacionada con menores niveles de bienestar psicológico (Silva et al., 2016).

Ahora bien, en el estudio realizado por Yáñez y Cárdenas (2010), tanto bolivianos como peruanos y colombianos presentaron niveles altos de bienestar psicológico, sin diferencias por género; mientras que Urzúa et al. (2018) dan cuenta de indicadores más altos de bienestar psicológico y de autoestima en colombianos comparados con peruanos.

$\mathrm{Y}$, finalmente, en cuanto al bienestar social, el estudio cuantitativo realizado en Chile por Urzúa, Delgado et al. (2017), informa niveles similares para peruanos y colombianos en las dimensiones "actualización social", "aceptación social" e "integración social", aunque en la dimensión "contribución social" los colombianos obtuvieron puntuaciones significativamente más altas que los peruanos, y estos últimos más altas que los colombianos en "coherencia social"; en general, solo en la dimensión "actualización social" los hombres obtuvieron puntuaciones significativamente superiores a las de las mujeres.

\section{Sintesis e hipótesis}

Como objetivos de la presente investigación se tuvo valorar y comparar - determinar diferencias de mediaslos indicadores de salud (SF-12) y de bienestar social entre chilenos e inmigrantes residentes en Chile, así como dar cuenta de las variables explicativas de los "niveles de salud" y del "bienestar social".

En cuanto a las hipótesis, tomando como base la "hipótesis selectiva de la inmigración", se esperaba que existieran mayores niveles de salud y bienestar social en inmigrantes que en los chilenos; $y$, respecto a las variables explicativas de la salud y del bienestar social, se esperaba que las variables socioculturales de "identidad endogrupal", "enriquecimiento cultural" y "cercanía intergrupal" tuvieran una relación significativa y positiva, mientras que la variable "percepción de discriminación" presentara una relación negativa.

A modo de síntesis, es importante señalar que este estudio es relevante en tanto (a) no se han publicado investigaciones en Chile en las que se comparen las puntuaciones obtenidas en estos constructos entre población chilena e inmigrante; (b) por los acotados estudios que dan cuenta del grupo de inmigrantes venezolanos, recientemente convertidos en el principal colectivo de inmigrantes residentes en Chile; y (c) por la escasa investigación de corte cuantitativo relacionada con indicadores de bienestar social y salud en muestras con población inmigrante en Chile y en Latinoamérica.

\section{Método}

\section{Tipo de estudio}

Se utilizó un diseño transversal de tipo descriptivo, correlacional y analítico, según los criterios de León y Montero (2015). 


\section{Procedimiento}

Inicialmente, encuestadores debidamente capacitados realizaron la recolección de datos, para lo cual se aplicó, de manera presencial, una encuesta en el lugar de trabajo del participante o en el lugar sugerido por él o ella, cuidando que el lugar cumpliera con las condiciones adecuadas para ello. Previo a la aplicación del cuestionario, se les explicaba brevemente a los participantes el propósito de la investigación, y, a quien accediera a participar, se le solicitaba que leyera y en caso de estar de acuerdo firmara el consentimiento informado. En este documento se contemplaba la confidencialidad de los datos, el carácter voluntario de la participación y la posibilidad de retirarse en cualquier momento sin tener consecuencia alguna. Ninguno de los participantes manifestó interés por retirarse durante el proceso.

\section{Participantes}

Los datos se recopilaron como parte de un proyecto más amplio sobre inmigración, salud e interculturalidad en Chile. La muestra recogida en Chile fue de tipo no probabilística por cuotas, y esta se equiparó por género - hombre y mujer - nacionalidad — chilenos, venezolanos, peruanos y colombianos - y lugar de residencia - región de Antofagasta, Valparaíso y Santiago, en el caso de los inmigrantes; y Región de Valparaíso y Santiago, en el caso de los chilenos-.

En particular, la muestra final estuvo compuesta por 155 chilenos $\left(M_{\text {edad }}=40.68, D T=14.85 ; 53.5 \%\right.$ mujeres $), 166$ colombianos $\left(M_{\text {edad }}=34.51, D T=11.54 ; 57.8 \%\right.$ mujeres $)$, 180 venezolanos $\left(M_{\text {edad }}=33.84, D T=10.38 ; 51.1 \%\right.$ mujeres), y 154 peruanos $\left(M_{\text {edad }}=33.55, D T=10.90 ; 49.35 \%\right.$ mujeres). Como criterio de inclusión se consideró a personas de 18 o más años, de nacionalidad chilena, colombiana, venezolana o peruana; $y$, en el caso de las personas extranjeras, se excluyó a quienes llevaran residiendo menos de seis meses en Chile o que tuvieran doble nacionalidad (véase Tabla 1).

\section{Instrumentos}

Se incluyeron diversas medidas sociodemográficas, junto con otras dirigidas a evaluar la salud y el bienestar social de los miembros de cada uno de los grupos, tal como se describe a continuación.

\section{Salud fisica y mental}

Para medir el nivel de salud de los participantes se utilizó la versión abreviada de la Escala de salud física y mental (SF-12) propuesta por Ware et al. (1996); escala que ha sido validada transculturalmente en estudios previos, $\mathrm{y}$ cuyos resultados dan cuenta de correlaciones superiores a $r=.94$ entre la versión abreviada (SF-12) y la completa (SF-36) (Gandek et al., 1998), y valores alfa de Cronbach por dimensión superiores a .74 en la versión validada en Chile (Vera-Villarroel et al., 2014). Específicamente, la escala consta de una dimensión de salud general (1 ítem), una de salud mental (6 ítems), y una de salud física (4 ítems). Los valores alfa de Cronbach obtenidos en la presente investigación fueron de $\alpha_{\text {chilenos }}=.85 \mathrm{y} \alpha_{\text {inmigrantes }}=.75$.

\section{Bienestar social}

Se utilizó la versión adaptada al español por Blanco y Díaz (2005) de la Escala de bienestar social propuesta por Keyes (1998), que consta de cinco dimensiones - integración social, aceptación social, contribución social, actualización social y coherencia social- Esta escala ha sido utilizada en diferentes contextos, en los cuales la versión adaptada al español presenta una adecuada consistencia interna, con indicadores que oscilan entre .68 y .83 (Blanco \& Díaz, 2005). Para esta investigación, se redujeron de 18 a 6 los ítems redactados de manera inversa para evitar los problemas psicométricos descritos cuando esta condición está presente (Van Sonderen et al., 2013). Los valores alfa de Cronbach obtenidos en la presente investigación fueron de $\alpha_{\text {chilenos }}=.81 \mathrm{y} \alpha_{\text {inmigrantes }}=.78$.

\section{Percepción de discriminación}

Para medir percepción de discriminación se utilizaron los ítems del Cuestionario de inmigrantes elaborado por Navas et al. (2004). El encabezado de la pregunta decía “¿En qué medida cree usted que los inmigrantes de su país son discriminados en Chile (se les trata peor que a los chilenos) en los siguientes aspectos?". Los participantes debían responder dentro de un rango de 1 ("Nada") a 5 ("Mucho") en los siguientes ítems: "en sus condiciones laborales", "en el alquiler de viviendas", "en bares, pubs o discotecas", "por su apariencia física", y "por su forma de hablar". Estos ítems solo se aplicaron a los participantes inmigrantes $(\alpha=.84)$.

\section{Identificación endogrupal}

La identificación endogrupal se evaluó con la pregunta “¿En qué grado se siente usted orgulloso de ser...?”, donde cada participante reemplazaba los puntos suspensivos por su respectiva nacionalidad. La puntuación fluctuaba entre 1 ("Nada") y 5 ("Mucho"). Este ítem se adaptó de la Escala para medir la identidad social —ítem de la subescala del ámbito privado - propuesta inicialmente por Luhtanen y 
Tabla 1.

Datos sociodemográficos y caracterización de chilenos e inmigrantes residentes en Chile

\begin{tabular}{|c|c|c|c|c|c|c|c|c|}
\hline & \multicolumn{2}{|c|}{ Chilenos } & \multicolumn{2}{|c|}{ Peruanos } & \multicolumn{2}{|c|}{ Colombianos } & \multicolumn{2}{|c|}{ Venezolanos } \\
\hline & $\begin{array}{c}\text { Hombres } \\
(\mathrm{n}=72)\end{array}$ & $\begin{array}{l}\text { Mujeres } \\
(\mathrm{n}=83)\end{array}$ & $\begin{array}{c}\text { Hombres } \\
(\mathrm{n}=78)\end{array}$ & $\begin{array}{c}\text { Mujeres } \\
(\mathrm{n}=76)\end{array}$ & $\begin{array}{l}\text { Hombres } \\
(\mathrm{n}=70)\end{array}$ & $\begin{array}{l}\text { Mujeres } \\
(\mathrm{n}=96)\end{array}$ & $\begin{array}{c}\text { Hombres } \\
(\mathrm{n}=88)\end{array}$ & $\begin{array}{r}\text { Mujeres } \\
(\mathrm{n}=92)\end{array}$ \\
\hline \multicolumn{9}{|l|}{ Edad } \\
\hline Media & 41.08 & 40.33 & 31.72 & 35.30 & 32.47 & 36.02 & 34.11 & 33.70 \\
\hline$D T^{*}$ & $(14.90)$ & $(14.89)$ & $(9.51)$ & $(11.97)$ & $(11.72)$ & $(11.22)$ & $(8.52)$ & $(11.93)$ \\
\hline \multicolumn{9}{|l|}{ Nivel educativo (\%) } \\
\hline Bajo & 31.9 & 30.1 & 56.4 & 56.6 & 49.3 & 52.1 & 27.3 & 15.4 \\
\hline Medio & 33.4 & 28.9 & 33.3 & 32.9 & 29.0 & 33.3 & 31.8 & 37.3 \\
\hline Alto & 34.7 & 41.0 & 10.3 & 10.5 & 21.7 & 14.6 & 40.9 & 47.3 \\
\hline \multicolumn{9}{|l|}{ Identificación étnica (\%) } \\
\hline Afroamericano & 1.4 & 0.0 & 0.0 & 1.4 & 4.4 & 15.1 & 7.1 & 7.8 \\
\hline Indígena & 7.0 & 4.8 & 10.3 & 6.8 & 0.0 & 0.0 & 1.2 & 4.5 \\
\hline Mestizo & 58.3 & 62.7 & 79.4 & 82.4 & 82.4 & 69.8 & 64.7 & 63.3 \\
\hline Blanco & 33.3 & 32.5 & 10.3 & 9.4 & 13.2 & 15.1 & 27.0 & 24.4 \\
\hline \multicolumn{9}{|l|}{ Estado civil (\%) } \\
\hline Soltero & 54.2 & 43.4 & 61.5 & 46.1 & 53.6 & 54.2 & 51.1 & 63.3 \\
\hline Casado/conviviente & 34.7 & 45.8 & 32.1 & 46.1 & 42.1 & 35.4 & 45.5 & 25.6 \\
\hline Separado/divorciado & 8.3 & 8.4 & 6.4 & 5.3 & 2.9 & 8.3 & 2.3 & 10.0 \\
\hline Viudo & 2.8 & 2.4 & 0.0 & 2.5 & 1.4 & 2.1 & 1.1 & 1.1 \\
\hline \multicolumn{9}{|l|}{ Hijos (\%) } \\
\hline No & 41.7 & 37.3 & 50 & 28.9 & 47.1 & 37.5 & 51.1 & 51.1 \\
\hline Sí & 58.3 & 62.7 & 50 & 71.1 & 52.9 & 62.5 & 48.9 & 48.9 \\
\hline \multicolumn{9}{|l|}{ Región de residencia } \\
\hline Antofagasta & & & 19.2 & 25.0 & 30.0 & 37.4 & 29.5 & 25.0 \\
\hline Valparaíso & 54.2 & 44.6 & 39.8 & 25.0 & 37.1 & 31.3 & 52.3 & 42.4 \\
\hline Santiago & 45.8 & 55.4 & 41.0 & 50.0 & 32.9 & 31.3 & 18.2 & 32.6 \\
\hline \multicolumn{9}{|l|}{$\begin{array}{l}\text { Tiempo residiendo en } \\
\text { Chile (\%) }\end{array}$} \\
\hline$\geq 6 \leq 12$ meses & & & 17.9 & 3.9 & 21.4 & 16.7 & 45.5 & 47.8 \\
\hline$>12 \leq 24$ meses & & & 15.4 & 21.1 & 31.5 & 22.9 & 29.5 & 35.9 \\
\hline$>24$ meses & & & 66.7 & 75.0 & 47.1 & 60.4 & 25.0 & 16.3 \\
\hline \multicolumn{9}{|l|}{ Ingresos $* *(\%)$} \\
\hline$\leq 560$ & & & 41.6 & 57.9 & 44.3 & 58.3 & 35.6 & 44.4 \\
\hline$>561 \leq 850$ & & & 33.7 & 25.0 & 27.1 & 19.8 & 31.1 & 31.1 \\
\hline$>851 \leq 1.400$ & & & 14.3 & 6.6 & 17.2 & 10.4 & 14.9 & 12.2 \\
\hline$>1401 \leq 2.110$ & & & 6.5 & 2.6 & 5.7 & 4.2 & 9.2 & 6.7 \\
\hline$>2.110$ & & & 3.9 & 7.9 & 5.7 & 7.3 & 9.2 & 5.6 \\
\hline \multicolumn{9}{|l|}{ Discriminación } \\
\hline Media $(D T)$ & & & $2.63(1.16)$ & $2.57(1.19)$ & $2.42(1.13)$ & $\begin{array}{c}2.77 \\
(1.02)\end{array}$ & $2.25(0.95)$ & $\begin{array}{l}2.53 \\
(0.99)\end{array}$ \\
\hline \multicolumn{9}{|l|}{ Enriquecimiento cultural } \\
\hline Media $(D T)$ & & & $2.78(0.47)$ & $2.93(0.47)$ & $2.86(0.52)$ & $\begin{array}{c}2.77 \\
(0.50)\end{array}$ & $2.92(0.44)$ & $\begin{array}{c}2.87 \\
(0.50)\end{array}$ \\
\hline \multicolumn{9}{|l|}{ Identidad endogrupal } \\
\hline Media $(D T)$ & & & $4.45(0.82)$ & $4.49(0.81)$ & $4.54(0.77)$ & $\begin{array}{c}4.61 \\
(0.64)\end{array}$ & $4.57(0.73)$ & $\begin{array}{c}4.57 \\
(0.72)\end{array}$ \\
\hline \multicolumn{9}{|l|}{ Cercanía intergrupal } \\
\hline Media $(D T)$ & & & $3.22(0.89)$ & $2.96(0.97)$ & $3.00(1.17)$ & $\begin{array}{l}2.76 \\
(0.95)\end{array}$ & $3.01(0.98)$ & $\begin{array}{c}2.71 \\
(1.09)\end{array}$ \\
\hline
\end{tabular}

Nota. ${ }^{*} D T=$ desviación típica; $* *$ moneda expresada en dólares americanos (US\$). 
Crocker (1992), y que ha sido incluido, con ciertas modificaciones, en distintas mediciones de identidad grupal (Cameron, 2004; Ellemers et al., 1999; Jackson, 2002; Navas et al., 2004).

\section{Cercanía intergrupal}

Para medir el grado de cercanía o lejanía intergrupal percibida por los participantes de los tres grupos de inmigrantes se diseñó la pregunta "¿Qué dibujo crees que representa mejor la relación entre tú y los chilenos?", donde se debía marcar uno de los cinco dibujos estructurados cada uno como dos conjuntos que iban de la nula a la máxima interacción. Este ítem se adaptó de la medida de intimidad intergrupal propuesta por Turner y Feddes (2011), que consta originalmente de tres ítems.

\section{Enriquecimiento cultural}

Para medir el grado de enriquecimiento cultural se utilizó la medida propuesta por Navas et al. (2004), ¿Cómo cree usted que es la influencia de la cultura chilena sobre su cultura?"; un ítem que consideraba cuatro opciones de respuesta que fluctúan entre 1 ("Muy mala") y 4 ("Muy buena").

\section{Sentimientos de felicidad y soledad}

Los sentimientos de felicidad y soledad se evaluaron con la medida de ítem único propuesta por Wold (1995). El encabezado del ítem de felicidad era "En general, ¿cómo se siente usted en su vida actual?", y comprendía un rango de respuesta de 1 ("No soy nada feliz") a 4 ("Completamente feliz"); mientras que el ítem de soledad, "¿Se siente solo alguna vez?", tenía un rango de respuesta de 1 ("Nunca me siento solo/a") a 3 ("A menudo me siento solo/a"). Estas preguntas fueron utilizadas para contrastar la validez concurrente y discriminante de las variables de salud y bienestar social.

\section{Análisis estadístico}

El análisis estadístico se realizó con el programa informático IBM SPSS Statistics, v. 25. Inicialmente, se realizaron análisis descriptivos - tendencia central y dispersión-y análisis de correlaciones $-r$ de Pearson - para dar cuenta de la relación existente entre las variables de salud (SF-12), bienestar social, sentimiento de felicidad y de soledad. Posteriormente, se utilizó la prueba $t$ para comparar el grupo de chilenos con el de inmigrantes, y se realizaron análisis de varianza (ANOVA) con el propósito de comprobar las diferencias de medias entre los grupos en los indicadores de salud (SF-12) y bienestar social. A continuación, se estimó una prueba posthoc — el estadístico Games Howell — para determinar las diferencias específicas por grupos.

Finalmente, previa comprobación del cumplimiento de los supuestos, se realizó un análisis de regresión jerárquico multivariante con la muestra de inmigrantes para dar cuenta de las variables predictoras del bienestar social y de salud, y se construyeron dos modelos para cada caso: en el primero se incluyeron las variables sociodemográficas a modo de control, y en el segundo se incluyeron las variables socioculturales de "percepción de discriminación", "identificación endogrupal", "cercanía intergrupal" y "enriquecimiento cultural". Específicamente, se chequearon y respetaron los supuestos de homocedasticidad - la relación entre pronósticos y residuos-, independencia — con el estadístico de Durbin-Watson: $\geq 1.5 \leq 2.5$ (Draper \& Smith, 1998)-, normalidad - histogramas con curva de normalidad, y de no colinealidad - tolerancia $>.10$; factor de inflación de la varianza $<10$ (Pardo et al., 2015) - . Los resultados de las pruebas estadísticas se consideraron significativos al nivel de probabilidad del $5 \%(p \leq .05)$.

\section{Consideraciones éticas}

La investigación fue evaluada y aprobada por el Comité ético y científico de la Universidad de Valparaíso (Acta de aprobación bioética CEC 142-17).

\section{Resultados}

En la Tabla 2 se presenta la matriz de correlaciones $(r$ de Pearson) de las variables. De acuerdo con lo esperado, todas las variables presentaron correlaciones significativas que oscilaron en valor absoluto entre $r=.167 \mathrm{y} r=.473$ $(p<.01)$. En particular, es importante destacar que la variable "sentimiento de soledad" presentó correlaciones significativas con coeficientes negativos con el "nivel de salud" (SF-12), el "bienestar social" y el "sentimiento de felicidad" $(p<.01)$.

Tabla 2.

Correlaciones bivariadas entre las variables

\begin{tabular}{lcccc}
\hline & 1 & 2 & 3 & 4 \\
\hline 1. SF-12 & & .264 & .298 & -.331 \\
2. Bienestar social & .376 & & .167 & -.173 \\
3. Sentimiento de felicidad & .465 & .421 & & -.434 \\
4. Sentimiento de soledad & -.473 & -.221 & -.422 & \\
\hline
\end{tabular}

Nota. Debajo de la diagonal se informan las correlaciones para la muestra de chilenos $(n=155)$, y por sobre la diagonal, la de inmigrantes $(n=500)$. Todas las correlaciones fueron significativas al nivel de $p<.01$. 
Asimismo, al comparar los niveles de salud entre chilenos e inmigrantes, se encuentra una diferencia estadísticamente significativa entre sus valores medios $t(203.579)=-2.745$, $p<.01$, donde el grupo de inmigrantes muestra una media superior en salud $(M=40.35, D T=4.44)$ que la de los chilenos $(M=38.93, D T=5.79)$. También, se encuentran diferencias significativas a favor de los inmigrantes en el bienestar social $\left(M_{\text {chilenos }}=85.42, D T=10.75 ; M_{\text {inmigrantes }}=88.62\right.$, $D T=8.57 ; t(190.778)=-3.197, p<.01$.

Por otra parte, se utilizó el procedimiento estadístico de análisis de varianza (ANOVA) con el propósito de comprobar las diferencias de medias entre los grupos (véase Tabla 3 ) en las variables "nivel de salud" (SF-12) y "bienestar social". Previo a ello, se analizaron las pruebas de normalidad por medio del test de Kolmogorov-Smirnov (K-S), y la prueba de homogeneidad de varianza con la prueba de Levenne. Tanto para la escala de salud SF-12 (K-S = .076; $p \leq .01)$ como para la de bienestar social $(\mathrm{K}-\mathrm{S}=.103 ; p \leq .01) \mathrm{se}$ rechazó la hipótesis de la distribución normal de los datos.

Estos resultados eran esperables, ya que este estadístico es especialmente sensible a pequeñas desviaciones de la normalidad con muestras grandes (Pardo et al., 2015). Dada esta condición, se decidió seguir la estrategia planteada por Curran et al. (1996) con muestras grandes, según la cual se interpreta el punto de corte problemático de los valores absolutos del estadístico de asimetría $(> \pm 2.0$, ) y de la curtosis $(> \pm 7.0)$. De acuerdo con este criterio, los valores obtenidos en la escala de salud (asimetría $=-.489$; curtosis $=.340$ ) como en la de bienestar social (asimetría $=.041$; curtosis $=1.52$ ) se relacionaron con pequeñas desviaciones de la normalidad, aunque estas no afectan el análisis de los datos.

Ahora bien, de acuerdo con los resultados de la prueba de Levenne, no se cumple la hipótesis de homogeneidad de varianzas para la escala de salud (SF-12) (Levenne 3, $619=3.435, p=.017$ ) como tampoco para la del bienestar social (Levenne 3, 590 = 5.314, $p<.01$ ). Atendiendo a ello, se utilizó para las pruebas posthoc el estimador GamesHowell (Pardo et al., 2015).

Por su parte, los resultados del ANOva muestran que las diferencias entre los grupos no resultaron significativas en "sentimiento de felicidad" $\left(F_{(3,652)}=1.366, p=.25\right) \mathrm{ni}$ en "sentimiento de soledad" $\left(F_{(3,651)}=2.408, p=.07\right)$; mientras que las variables que medían "salud" (SF-12; $F_{(3,619)}=3.345$, $p<.017 ; d=0.15)$ y "bienestar social" $\left(F_{(3,590)}=5.314\right.$, $p<.001 ; d=0.21)$ sí presentaron diferencias significativas entre los grupos. Para determinar las diferencias específicas según el grupo de pertenencia — chilenos, peruanos, colombianos y venezolanos-, se realizó una serie de análisis posthoc — con el estimador de Games-Howell —. Los resultados indican que entre chilenos y peruanos $(\Delta \bar{x}=-.798$; $p=.53)$ y entre chilenos y colombianos $(\Delta \bar{x}=-1.42 ; p=.83)$ no hay evidencia de diferencias significativas en el nivel de salud (SF-12); mientras que entre chilenos y venezolanos sí se observó una diferencia significativa a favor de estos últimos $(\Delta \bar{x}=-1.99 ; p=.005)$.

Entre los tres grupos de inmigrantes, no se encontraron diferencias significativas en sus niveles de salud. Ahora bien, en cuanto al "bienestar social", la muestra de venezolanos presentó niveles significativamente más altos que los de los chilenos $(\Delta \bar{x}=0.22 ; p<.001)$, colombianos $(\Delta \bar{x}=0.11$; $p<.026)$ y peruanos $(\Delta \bar{x}=017 ; p<.001)$. Aunque los chilenos presentaron niveles de bienestar social más bajos que los de los colombianos $(\Delta \bar{x}=-0.11 ; p=.114)$ y los peruanos $(\Delta \bar{x}=-0.05 ; p<.699)$; estas diferencias no fueron estadísticamente significativas.

A continuación, el análisis de regresión múltiple utilizado para predecir las variables explicativas de la salud y el bienestar social en los grupos de inmigrantes se presenta en la Tabla 4. Una mirada a las variables sociodemográficas (Modelo 1) permite concluir que los hombres inmigrantes tienen mayores niveles de salud que las mujeres inmigrantes $(\beta=.13, p<.01)$, y que el "nivel de estudios" tiene una relación positiva con la salud $(\beta=.14, p<.01)$. Sin embargo, al introducir en el modelo las variables socioculturales (Modelo 2), además de continuar siendo significativas las variables "género" y "nivel de estudios" en la explicación del nivel de salud, se suma a estas la variable "nivel de ingresos" $(\beta=.12, p<.05)$. La única variable sociocultural con un efecto significativo en la salud de los inmigrantes fue la "percepción de discriminación" $(\beta=-.24, p<.001)$, lo que demuestra que, a mayor percepción de discriminación, menor nivel de salud de los inmigrantes.

Ahora bien, los resultados del Modelo 3 indican que el "nivel de estudios" se relaciona con un mayor nivel de "bienestar social" $(\beta=.22, p<.001)$, y, en concordancia con el Modelo 1 (Salud, SF-12), las mujeres también muestran menores niveles que los hombres en la variable "bienestar social".

Finalmente, al incluir las variables sociodemográficas (Modelo 4), se encuentra que la "identificación endogrupal" ( $\beta=.12, p<.01)$, la "cercanía intergrupal" $(\beta=.26, p<.001)$ $\mathrm{y}$ el "enriquecimiento cultural" $(\beta=.16, p<.001)$ tuvieron efectos significativos y positivos sobre el "bienestar social".

\section{Discusión}

En este estudio se examinaron y compararon los niveles de "salud" (SF-12) y de "bienestar social" (Keyes, 1998) en una muestra de chilenos e inmigrantes de nacionalidad colombiana, venezolana y peruana, residentes en la zona 
Tabla 3.

Comparaciones posthoc entre grupos en las variables de salud fisica y mental (SF-12) y bienestar social

\begin{tabular}{|c|c|c|c|c|c|}
\hline Variable & Grupo & Grupo & $\begin{array}{l}\text { Diferencias de } \\
\text { medias } \\
\text { (error estándar) }\end{array}$ & IC $95 \%$ & $\begin{array}{c}\text { Tamaño del } \\
\text { efecto }(d)\end{array}$ \\
\hline \multirow{9}{*}{ SF-12 } & \multirow{5}{*}{$\begin{array}{c}\text { Chilenos } \\
(M=38.93 ; D T=5.79)\end{array}$} & & & \multirow{2}{*}{$-2.33 ; 0.73$} & \multirow{4}{*}{0.14} \\
\hline & & $(M=39.72 ; D T=4.34)$ & $(0.59)$ & & \\
\hline & & Colombianos & -1.42 & \multirow{2}{*}{$-2.95 ; 0.12$} & \\
\hline & & $(M=40.34 ; D T=4.44)$ & $(0.60)$ & & \\
\hline & & $\begin{array}{c}\text { Venezolanos } \\
(M=40.91 ; D T=4.48)\end{array}$ & $\begin{array}{r}-1.99 * \\
(0.59) \\
\end{array}$ & $-3.50 ;-0.47$ & 0.20 \\
\hline & \multirow{3}{*}{ Peruanos } & Colombianos & -0.62 & \multirow[t]{2}{*}{$-1.92 ; 0.68$} & \\
\hline & & & $(0.50)$ & & \\
\hline & & Venezolanos & $\begin{array}{l}-1.19 \\
(0.49)\end{array}$ & $-2.46 ; 0.08$ & \\
\hline & Colombianos & Venezolanos & $\begin{array}{l}-0.57 \\
(0.50) \\
\end{array}$ & $-1.85 ; 0.72$ & \\
\hline \multirow{9}{*}{$\begin{array}{l}\text { Bienestar } \\
\text { social }\end{array}$} & \multirow{5}{*}{$\begin{array}{c}\text { Chilenos } \\
(M=3.42 ; D T=0.43)\end{array}$} & Peruanos & -0.05 & \multirow{2}{*}{$-0.17 ; 0.07$} & \\
\hline & & $(M=3.50 ; D T=0.33)$ & $(0.05)$ & & \\
\hline & & Colombianos & -0.11 & \multirow{2}{*}{$-0.23 ; 0.02$} & \\
\hline & & $(M=3.52 ; D T=0.36)$ & $(0.05)$ & & \\
\hline & & $\begin{array}{c}\text { Venezolanos } \\
(M=3.63 ; D T=0.32)\end{array}$ & $\begin{array}{c}-0.22 * * \\
(0.04)\end{array}$ & $-0.33 ;-0.10$ & 0.28 \\
\hline & \multirow{3}{*}{ Peruanos } & 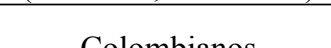 & -0.06 & \multirow{2}{*}{$-0.16 ; 0.05$} & \\
\hline & & Colombianos & $(0.04)$ & & \\
\hline & & Venezolanos & $\begin{array}{c}-0.17 * * \\
(0.04)\end{array}$ & $-0.26 ;-0.07$ & 0.20 \\
\hline & Colombianos & Venezolanos & $\begin{array}{l}-0.11 * \\
(0.04) \\
\end{array}$ & $-0.21 ;-0.01$ & 0.16 \\
\hline
\end{tabular}

Nota. Se reportan solo los tamaños del efecto $(d)$ de las diferencias de medias que fueron significativas. ${ }^{*} p<.05 ; * * p<.001$.

Tabla 4.

Coeficientes de regresión de variables predictoras de la salud (SF-12) y el bienestar social en personas inmigrantes residentes en Chile

\begin{tabular}{|c|c|c|c|c|c|c|c|c|c|c|c|c|}
\hline & \multicolumn{6}{|c|}{ Salud (SF-12) } & \multicolumn{6}{|c|}{ Bienestar social } \\
\hline & \multicolumn{3}{|c|}{ Modelo 1} & \multicolumn{3}{|c|}{ Modelo 2} & \multicolumn{3}{|c|}{ Modelo 3} & \multicolumn{3}{|c|}{ Modelo 4} \\
\hline & $\mathrm{b}$ & $E T$ & $\beta$ & $\mathrm{b}$ & $E T$ & $\beta$ & $\mathrm{b}$ & $E T$ & $\beta$ & $\mathrm{b}$ & $E T$ & $\beta$ \\
\hline Constante & $39.47 * * *$ & 1.50 & & $37.76^{* * *}$ & 2.68 & & $3.17 * * *$ & 0.12 & & $2.30 *$ & 0.20 & \\
\hline Género $^{1}$ & $1.16^{* *}$ & 0.42 & $0.13 * *$ & $0.85^{*}$ & 0.41 & $0.10^{*}$ & $0.08 *$ & 0.03 & $0.11 *$ & $0.07^{*}$ & 0.03 & $0.10^{*}$ \\
\hline Edad & -0.03 & 0.02 & -0.07 & -0.03 & 0.02 & -0.07 & 0.00 & 0.00 & 0.10 & 0.00 & 0.00 & 0.07 \\
\hline Estudios & $0.33 * *$ & 0.12 & $0.14^{* *}$ & $0.29 *$ & 0.12 & $0.12 * *$ & $0.04 * * *$ & 0.01 & $0.22 * * *$ & $0.04 *$ & 0.01 & $0.24 *$ \\
\hline Ingresos & 0.28 & 0.18 & 0.08 & $0.40 *$ & 0.18 & $0.12 *$ & 0.02 & 0.01 & 0.10 & 0.02 & 0.01 & 0.06 \\
\hline Hijos & 0.35 & 0.48 & 0.04 & 0.41 & 0.47 & 0.05 & 0.06 & 0.04 & 0.09 & 0.06 & 0.04 & 0.08 \\
\hline Tiempo en Chile & 0.00 & 0.00 & -0.02 & 0.00 & 0.00 & 0.01 & 0.00 & 0.00 & 0.00 & 0.00 & 0.00 & -0.02 \\
\hline Amistades previas en Chile & -0.58 & 0.43 & -0.06 & -0.24 & 0.42 & -0.03 & $-0.07 *$ & 0.03 & -0.09 & -0.06 & 0.03 & -0.08 \\
\hline Percepción de discriminación & & & & $-1.01 * * *$ & 0.20 & $-0.24 * * *$ & & & & 0.00 & 0.02 & 0.01 \\
\hline Identificación endogrupal & & & & 0.09 & 0.33 & 0.01 & & & & $0.07 * *$ & 0.03 & $0.12 * *$ \\
\hline Cercanía intergrupal & & & & 0.32 & 0.21 & 0.07 & & & & $0.09 * * *$ & 0.02 & $0.26 * * *$ \\
\hline Enriquecimiento cultural & & & & 0.69 & 0.42 & 0.07 & & & & $0.11 * * *$ & 0.03 & $0.16^{* * *}$ \\
\hline \multicolumn{13}{|l|}{ Resumen del modelo } \\
\hline$R^{2}$ & \multicolumn{3}{|c|}{0.07} & \multicolumn{3}{|c|}{0.15} & \multicolumn{3}{|c|}{0.108} & \multicolumn{3}{|c|}{0.218} \\
\hline$F$ & \multirow{2}{*}{\multicolumn{3}{|c|}{$4.858 * * *$}} & \multicolumn{3}{|c|}{$6.738 * * *$} & \multirow{2}{*}{\multicolumn{3}{|c|}{$7.332 * * *$}} & \multicolumn{3}{|c|}{$10.666^{* * *}$} \\
\hline Cambio en $F$ & & & & \multicolumn{3}{|c|}{$9.378 * * *$} & & & & \multicolumn{3}{|c|}{$14.826 * * *$} \\
\hline
\end{tabular}

Nota. ${ }^{1} 0=$ Mujer; $1=$ Hombre. ${ }^{*} p<.05 ; * * p<.01 ; * * * p<.001$. 
norte y centro de Chile. Posteriormente, se indagó en torno a las variables explicativas de la salud y del bienestar social en el grupo de inmigrantes. En general, los resultados indican que se corrobora la "hipótesis selectiva de la inmigración", ya que los niveles de salud y de bienestar reportados por los inmigrantes fueron mayores a los de los chilenos.

También se encontró apoyo parcial para las variables socioculturales planteadas como predictoras de la "salud" y del "bienestar social". Específicamente, la "percepción de discriminación" fue la única variable sociocultural que mostró tener un efecto negativo en la salud, lo cual es coherente con los resultados reportados en la literatura (Mera-Lemp et al., 2019; Williams \& Mohammed, 2009). Asimismo, en el "bienestar social" fue más evidente el peso que tienen las variables socioculturales, pues tres de las variables planteadas — “identidad endogrupal", "enriquecimiento cultural" y "cercanía intergrupal" - mostraron una relación positiva con el "bienestar social".

Por otra parte, los resultados obtenidos dan cuenta de varios aspectos relevantes. En primer lugar, que los chilenos presentan niveles iguales o más bajos que los inmigrantes en las medidas de salud y bienestar social; indicadores compatibles con el fenómeno descrito en algunos estudios en Europa y EE. UU., donde los colectivos de inmigrantes, especialmente durante el primer tiempo de residencia en el país de acogida, presentan mejores indicadores de salud y de bienestar que el de la población autóctona - el fenómeno denominado en EE. UU. como "la paradoja de la salud hispana"-. Estos indicadores, conforme pasa el tiempo de residencia, se irían igualando o incluso empeorando en comparación con el de los nativos.

En la presente investigación, el "tiempo de residencia en Chile" no explica cambios en el "bienestar social", lo que podría deberse a que el rango del tiempo de residencia no ha sido lo suficientemente extenso como para que los inmigrantes hayan asimilado las condiciones de vida de los chilenos, a lo que se suma - en el caso de peruanos y colombianos-la "identidad endogrupal" que les permite vincularse a la sociedad de acogida desde una estrategia integradora - valorando y conservando elementos de su propia cultura e integrando elementos de la cultura de acogida-, lo que actúa como un factor protector. Complementariamente, y en sintonía con estudios internacionales (Bowden et al., 2016; Pettigrew et al., 2011), la red de apoyo social — medida por la "cercanía intergrupal" - opera como una de las variables más relevantes al momento de explicar el "bienestar social" en los inmigrantes. Y, por otra parte, en concordancia con los resultados de Murillo y Molero (2016) con medidas de bienestar subjetivo, la "percepción de discriminación" no tuvo un impacto en el bienestar social, como sí se observó en la medida objetiva de salud.
Finalmente, la variable "género" mostró de manera consistente que las mujeres inmigrantes suelen estar asociadas a un menor "nivel de salud" (SF-12) y de "bienestar social". Este resultado debe servir como un llamado de alarma para entender la realidad que experimentan las mujeres inmigrantes en Chile y los factores que podrían estar explicando sus menores niveles de salud y bienestar. A su vez, el "nivel de estudios" mostró también un efecto consistente en la salud y en el bienestar social. En ambos funciona como un factor facilitador el acceso de espacios sociales menos amenazantes y más proclives a las relaciones intergrupales positivas - p. ej., acceso a mejores empleos y servicios educativos o sanitarios-.

A modo de síntesis, los resultados dan cuenta del crucial efecto de la cercanía intergrupal en el bienestar social de las personas inmigrantes y en el negativo efecto de la discriminación grupal percibida en los indicadores de salud; hallazgos que confirman los beneficios del contacto intergrupal bajo un estilo basado en la integración, en el que las personas presentan una valoración positiva tanto del endo como del exogrupo.

\section{Fortalezas, limitaciones y futuras investigaciones}

La mayor parte de los estudios de corte cuantitativo realizados en Chile han utilizado muestras de inmigrantes de nacionalidad peruana y colombiana, y en menor medida boliviana, y han sido recogidas principalmente en la zona norte del país (Región de Antofagasta). En este estudio, a la muestra de colombianos y peruanos se sumó la venezolana, actualmente el grupo más numeroso de inmigrantes que residen en Chile. Además, la muestra no solo se recogió en Antofagasta - ciudad atractiva para los inmigrantes por la oferta laboral ligada a la minería y servicios-, sino que se incluyó también la región de Valparaíso y la Metropolitana (capital de Chile). Complementariamente, se aplicaron las medidas de salud y bienestar social en chilenos para compararlas con la de los inmigrantes, lo cual no se había realizado hasta el momento.

Una segunda fortaleza se vincula con la valoración de la especificidad de cada uno de los tres grupos de inmigrantes estudiados, lo cual se evidencia en la estrategia utilizada para explicar los niveles de salud y de bienestar social. Esto es relevante, ya que no existe "una" migración, sino experiencias específicas fuertemente influidas por las características diferenciadoras de uno u otro grupo. Finalmente, este estudio es un aporte importante al acotado número de investigaciones sobre inmigración y bienestar en el contexto latinoamericano, caracterizado por una migración sur-sur entre ciudadanos de países con niveles de desarrollo económico medio o medio-bajo, pertenecientes 
a la región con mayor presencia de desigualdad social en el mundo (CEPAL, 2016).

Como en todas las investigaciones, son diversas las limitaciones y desafíos que se desprenden del trabajo realizado. Por ejemplo, el diseño, al ser de tipo transversal, no valora los cambios en los indicadores de salud y bienestar a lo largo del tiempo, por lo que sería de gran relevancia considerar el diseño de investigaciones de corte longitudinal. Además, en la muestra no se consideró la distinción entre migración forzada y voluntaria, lo cual podría tener efectos diferenciadores en el bienestar social y la salud física y mental de las personas que han inmigrado por esta condición (Abello et al., 2009). En este sentido, sería aconsejable que futuras investigaciones abordaran el efecto en estas variables en personas desplazadas por violencia sociopolítica.

Finalmente, por razones de carácter económico y técnicos — traducción de instrumentos o capacitación de encuestadores bilingües, por ejemplo- no se consideró en esta muestra a personas residentes en la zona sur de Chile ni a personas de origen haitiano, quienes actualmente representan el tercer grupo de inmigrantes con mayor presencia en Chile. Se recomienda, por tanto, la inclusión de esta población en futuros estudios.

\section{Referencias}

Abello, R., Amaris, M., Blanco, A., Madariaga, C., Manrique, K., Martínez, M., \& Turizo, Y. (2009). Bienestar y trauma en personas adultas desplazadas por la violencia política. Universitas Psychologica, 8(2), 455-470. http://www.scie lo.org.co/pdf/rups/v8n2/v8n2a13.pdf

Arancibia, H., Blanco, A., Ruiz, M. A., \& Cárdenas, M. (2016). RIVEC (rejection, intimacy, values, emotions, and culture) prejudice scale: An adaptation to the chilean context of the blatant and subtle prejudice scale. Journal of Pacific Rim Psychology, 10, 1-12. https://doi.org/10.1017/prp.2016.3

Berry, J. W. (2005). Acculturation: Living successfully in two cultures. International Journal of Intercultural Relations, 29(6), 697-712. https://doi.org/10.1016/j. ijintrel.2005.07.013

Blanco, A., \& Díaz, D. (2005). El bienestar social: su concepto y medición. Psicothema, 17(4), 582-589. http://www.psicothema.com/psicothema.asp?id=3149

Bobowik, M., Basabe, N., \& Páez, D. (2015). The bright side of migration: Hedonic, psychological, and social well-being in immigrants in Spain. Social science research, 51, 189-204. https://doi.org/10.1016/j.ssresearch.2014.09.011

Bowden, J., Voci, A., Al Ramiah, A., Tausch, N., Hughes, J., \& Hewstone, M. (2016). Building Trust in a Postconflict Society:An Integrative Model ofCross-group Friendship and
Intergroup Emotions. Journal of Conflict Resolution, 60(6), 1041-1070. https://doi.org/10.1177/0022002714564427

Cabieses, B., Chepo, M., Oyarte, M., Markkula, N., Bustos, P., Pedrero, V., \& Delgado, I. (2017). Brechas de desigualdad en salud en niños migrantes versus locales en Chile. Revista Chilena de Pediatría, 88(6), 707-716. https://doi. org/10.4067/S0370-41062017000600707

Cabieses, B., \& Oyarte, M. (2020). Acceso a salud en inmigrantes: Identificando brechas para la protección social en salud. Revista de Saúde Pública, 54, 20. https://doi.org/10.11606/ s1518-8787.2020054001501

Cameron, J. E. (2004). A Three-Factor Model of Social Identity. Self and Identity, 3(3), 239-262. https://doi. org/10.1080/13576500444000047

Canales, A. I. (2019). La inmigración contemporánea en Chile. Entre la diferenciación étnico-nacional y la desigualdad de clases. Papeles de Población, 25(100), 53-85. https://rppo blacion.uaemex.mx/article/view/12245

Cárdenas, M. (2006). «Y verás cómo quieren en Chile...»: Un estudio sobre el prejuicio hacia los inmigrantes bolivianos por parte de los jóvenes chilenos. Ultima Década, 14(24), 99124. https://doi.org/10.4067/S0718-22362006000100006

Cárdenas, M., Gómez, F., Méndez, L., \& Yáñez, S. (2011). Reporte de los Niveles de Prejuicio Sutil y Manifiesto Hacia los Inmigrantes Bolivianos y Análisis de su Relación con Variables Psicosociales. Psicoperspectivas, 10(1), 125-143. https://doi.org/10.5027/ psicoperspectivas-Vol10-Issue1-fulltext-134

Centros de Estudios de Opinión Ciudadana (CEOC). (2009). Prejuicio $y$ discriminación racial en Chile. CEOC, Universidad de Talca. http://www.cenem.utalca.cl/docs/pu blicaciones/Prejuicio_y_discriminacion_racial_en_Chile. pdf

CEPAL. (2016). La matriz de la desigualdad social en América Latina. Naciones Unidas. https:/www.cepal.org/es/ publicaciones/40668-la-matriz-la-desigualdad-social-ame rica-latina

Chiu, S., Redelmeier, D. A., Tolomiczenko, G., Kiss, A., \& Hwang, S. W. (2009). The health of homeless immigrants. Journal of Epidemiology \& Community Health, 63(11), 943-948. http://dx.doi.org/10.1136/jech.2009.088468

Cho, Y., Frisbie, W. P., Hummer, R. A., \& Rogers, R. G. (2004). Nativity, duration of residence, and the health of Hispanic adults in the United States. International Migration Review, 38(1), 184-211. https://doi.org/10.1111/j.1747-7379.2004. tb00193.x

Concha, N. L., \& Mansilla, M. Á. (2017). Pacientes ilegítimos: Acceso a la salud de los inmigrantes indocumentados en Chile. Salud Colectiva, 13, 507-520. https://doi. org/10.18294/sc.2017.1110

Crocker, J., Luhtanen, R., Blaine, B., \& Broadnax, S. (1994). Collective self-esteem and psychological well-being among White, Black, and Asian college students. Personality and 
Social Psychology Bulletin, 20(5), 503-513. https://doi. org/10.1177/0146167294205007

Curran, P. J., West, S. G., \& Finch, J. F. (1996). The robustness of test statistics to nonnormality and specification error in confirmatory factor analysis. Psychological Methods, 1(1), 16-29. https://doi.org/10.1037/1082-989X.1.1.16

Departamento de Extranjería y Migración (DEM). (2016). Migración en Chile 2005-2014. Ministerio del Interior y Seguridad Pública. https://www.extranjeria.gob.cl/ media/2016/02/Anuario-Estad\%C3\%ADstico-NacionalMigraci\%C3\%B3n-en-Chile-2005-2014.pdf

Departamento de Extranjería y Migración (DEM). (2019). Estimación de personas extranjeras residentes en Chile al 31 de diciembre 2018. Informe metodológico. Instituto Nacional de Estadísticas (INE). https://www.ine.cl/prensa/2020/03/12/seg\%C3\%BAn-estimaciones-la-cantidadde-personas-extranjeras-residentes-habituales-en-chilebordea-los-1-5-millones-al-31-de-diciembre-de-2019

Draper, N. R., \& Smith, H. (1998). Applied regression analysis (3. ${ }^{\mathrm{a}}$ ed). Wiley.

El Mostrador. (2019, febrero 2). Gobierno inicia proceso de expulsión de 12 mil migrantes que no cumplen requisitos. El Mostrador. https://www.elmostrador.cl/dia/2019/02/02/ gobierno-inicia-proceso-de-expulsion-de-12-mil-migran tes-que-no-cumplen-requisitos/

Ellemers, N., Kortekaas, P., \& Ouwerkerk, J. W. (1999). Self-categorisation, commitment to the group and group self-esteem as related but distinct aspects of social identity. European Journal of Social Psychology, 29(2-3), 371-389. https://doi.org/10.1002/(SICI)10990992(199903/05)29:2/3<371::AID-EJSP932>3.0.CO;2-U

Franzini, L., Ribble, J., \& Keddie, M. (2001). Understanding the Hispanic paradox. Ethnicity \& Disease, 11(3), 496-518. https://pubmed.ncbi.nlm.nih.gov/11572416/

Gandek, B., Ware, J. E., Aaronson, N. K., Apolone, G., Bjorner, J. B., Brazier, J. E., Bullinger, M., Kaasa, S., Leplege, A., Prieto, L., \& Sullivan, M. (1998). Cross-Validation of Item Selection and Scoring for the SF-12 Health Survey in Nine Countries: Results from the IQOLA Project. Journal of Clinical Epidemiology, 51(11), 1171-1178. https://doi. org/10.1016/S0895-4356(98)00109-7

Gissi, N., Pinto-Baleisan, C., Rodríguez, F., Gissi, N., PintoBaleisan, C., \& Rodríguez, F. (2019). Inmigración reciente de colombianos y colombianas en Chile. Sociedades plurales, imaginarios sociales y estereotipos. Estudios Atacameños, (62), 127-141. https:/doi.org/10.22199/ issn.0718-1043-2019-0011

Hennessy-Burt, T. E., Stoecklin-Marois, M. T., MenesesGonzález, F., \& Schenker, M. B. (2011). A pilot binational study of health behaviors and immigration. Journal of Immigrant and Minority Health, 13(6), 1142-1149. https:// doi.org/10.1007/s10903-010-9387-8

Hoffmann, C., McFarland, B. H., Kinzie, J. D., Bresler, L., Rakhlin, D., Wolf, S., \& Kovas, A. E. (2005). Psychometric properties of a Russian version of the SF-12 Health Survey in a refugee population. Comprehensive Psychiatry, 46(5), 390-397. https://doi.org/10.1016/j.comppsych.2004.12.002

Huynh, V. W., \& Fuligni, A. J. (2010). Discrimination hurts: The academic, psychological, and physical well-being of adolescents. Journal of Research on Adolescence, 20(4), 916-941. https://doi.org/10.1111/j.1532-7795.2010.00670.x

Instituto Nacional de Estadísticas (INE). (2018). Características de la inmigración internacional en Chile, Censo 2017. INE. http://www.censo2017.cl/descargas/inmigracion/181123documento-migracion.pdf

Instituto Nacional de Estadísticas (INE). (2019). Censos de Población Históricos. INE. https://www.ine.cl/esta disticas/sociales/censos-de-poblacion-y-vivienda/pobla cion-y-vivienda

Jackson, J. W. (2002). Intergroup Attitudes as a Function of Different Dimensions of Group Identification and Perceived Intergroup Conflict. Self and Identity, 1(1), 11-33. https:// doi.org/10.1080/152988602317232777

Jasinskaja-Lahti, I., Liebkind, K., Jaakkola, M., \& Reuter, A. (2006). Perceived discrimination, social support networks, and psychological well-being among three immigrant groups. Journal of Cross-Cultural Psychology, 37(3), 293311. https://doi.org/10.1177/0022022106286925

Keyes, C. (1998). Social well-being. Social Psychology Quarterly, 61(2), 121-140. https://doi.org/10.2307/2787065

Keyes, C. (2006). Subjective Well-Being in Mental Health and Human Development Research Worldwide: An Introduction. Social Indicators Research, 77(1), 1-10. https://doi.org/10.1007/s11205-005-5550-3

León, O., \& Montero, I. (2015). Métodos de investigación en psicología y educación: Las tradiciones cuantitativa y cualitativa (4. ${ }^{\mathrm{a}}$ ed.). McGraw-Hill.

Luhtanen, R., \& Crocker, J. (1992). A Collective Self-Esteem Scale: Self-Evaluation of One's Social Identity. Personality and Social Psychology Bulletin, 18(3), 302-318. https://doi. org $/ 10.1177 / 0146167292183006$

Márquez, F. (2014). Inmigrantes en territorios de frontera: La ciudad de los otros. Santiago de Chile. EURE, 40(120), 4972. http://dx.doi.org/10.4067/S0250-71612014000200003

Mera-Lemp, M. J., Martínez-Zelaya, G., Orellana, A., \& SmithCastro, V. (2020). Orientaciones de aculturación, estrés de aculturación y bienestar psicológico en inmigrantes latinoamericanos en Santiago de Chile. Acta Colombiana de Psicología, 23(1), 216-230. https://doi.org/10.14718/ ACP.2020.23.1.11

Mera-Lemp, M. J., Ramírez-Vielma, R., Bilbao, M. de los Á., \& Nazar, G. (2019). La discriminación percibida, la empleabilidad y el bienestar psicológico en los inmigrantes latinoamericanos en Chile. Revista de Psicología del Trabajo y de las Organizaciones, 35(3), 227-236. https:// doi.org/10.5093/jwop2019a24 
Murillo, J., \& Molero, F. (2016). Relación entre la Satisfacción Vital y otras Variables Psicosociales de Migrantes Colombianos en Estados Unidos. Revista Colombiana de Psicología, 25(1), 15-32. https://doi.org/10.15446/rcp. v25n 1.44308

Navas, L., \& Sánchez, A. (2010). Actitudes de los Estudiantes de Pedagogía de las Regiones del Bío Bío y la Araucanía de Chile hacia la Presencia de Niños Inmigrantes en la Escuela: Análisis Diferenciales. Psykhe, 19(1), 47-60. https://doi. org/10.4067/S0718-22282010000100004

Navas, M., Pablo, L., Fernández, P., Sánchez, J., M Carmen, M., Fernández, G., Rojas-Tejada, A., Cuadrado Guirado, I., Matilde, G., García, A., Fernández Prados, J., Prados, F., Marisol, N., Pumares, P., Sánchez Fernández, J., Miranda, M., Carmen, G., J Rojas Fernández, A., Cuadrado Tejada, I., \& Gobernación, D. (2004). Estrategias y actitudes de aculturación: La perspectiva de los inmigrantes y de los autóctonos en Almería. Universidad de Almería.

Nesterko, Y., Turrión, C. M., Friedrich, M., \& Glaesmer, H. (2019). Trajectories of health-related quality of life in immigrants and non-immigrants in Germany: A population-based longitudinal study. International Journal of Public Health, 64(1), 49-58. https://doi.org/10.1007/s00038-018-1113-7

Pardo, A., Ruiz, M. Á., \& San Martín, R. (2015). Análisis de datos en ciencias sociales y de la salud II. Síntesis.

Parker-Frisbie, W., Cho, Y., \& Hummer, R. A. (2001). Immigration and the health of Asian and Pacific Islander adults in the United States. American Journal of Epidemiology, 153(4), 372-380. https://doi.org/10.1093/ aje/153.4.372

Pascoe, E. A., \& Smart-Richman, L. (2009). Perceived discrimination and health: A meta-analytic review. Psychological Bulletin, 135(4), 531-554. https://doi.org/10.1037/ a0016059

Pavez-Soto, I., Ortiz-López, J., \& Domaica-Barrales, A. (2020). Percepciones de la comunidad educativa sobre estudiantes migrantes en Chile: Trato, diferencias e inclusión escolar. Estudios Pedagógicos, 45(3), 163-183. https://doi. org/10.4067/S0718-07052019000300163

Pettigrew, T. F., Tropp, L. R., Wagner, U., \& Christ, O. (2011). Recent advances in intergroup contact theory. International Journal of Intercultural Relations, 35(3), 271-280. https:// doi.org/10.1016/j.ijintrel.2011.03.001

Ramírez-Santana, M., Rivera-Humeres, J., Bernales-Silva, M., \& Cabieses-Valdés, B. (2019). Vulnerabilidad social y necesidades de salud de población inmigrante en el norte de Chile. Migraciones Internacionales, 10. https://doi. org/10.33679/rmi.v1i36.2005

Rojas, G., Fritsch, R., Castro, A., Guajardo, V., Torres, P., \& Díaz, B. (2011). Trastornos mentales comunes y uso de servicios de salud en población inmigrante. Revista Médica de Chile, 139(10), 1298-1304. https://doi.org/10.4067/ S0034-98872011001000008
Rojas, N., Amode, N., \& Vásquez, J. (2015). Racismo y matrices de "inclusión" de la migración haitiana en Chile: Elementos conceptuales y contextuales para la discusión. Polis. Revista Latinoamericana, 42, 1-24. http://dx.doi. org/10.4067/S0718-65682015000300011

Salas, N., Castillo, D., San Martín, C., Kong, F., Thayer, E., \& Huepe, D. (2017). Inmigración en la escuela: Caracterización del prejuicio hacia escolares migrantes en Chile. Universitas Psychologica, 16(5), 1-15. https://doi. org/10.11144/Javeriana.upsy16-5.iecp

Segovia, J. S., \& Lufin, M. (2013). Approaches to the AfroColombian experience in Chile: South-south immigration toward the northern regions. Journal of Black Studies, 44(3), 231-251. https://www.jstor.org/stable/23414669

Seligman, M. E., \& Csikszentmihalyi, M. (2000). Positive psychology: An introduction. American Psychologist, 55(1), 5-14. https://doi.org/10.1037//0003-066X.55.1.5

Sevillano, V., Basabe, N., Bobowik, M., \& Aierdi, X. (2014). Health-related quality of life, ethnicity and perceived discrimination among immigrants and natives in Spain. Ethnicity \& Health, 19(2), 178-197. https://doi.org/10.1080/1355785 8.2013.797569

Silva, J., Urzúa, A., Caqueo-Urizar, A., Lufin, M., \& Irarrazaval, M. (2016). Bienestar psicológico y estrategias de aculturación en inmigrantes afrocolombianos en el norte de Chile. Interciencia, 41(12), 804-811. http://www.redalyc.org/articu lo.oa? id=33948806002

Stang, M. F., \& Stefoni, C. (2016). La microfísica de las fronteras. Criminalización, racialización y expulsabilidad de los migrantes colombianos en Antofagasta, Chile. Astrolabio, 17, 42-80. https://revistas.unc.edu.ar/index.php/astrolabio/ article/view/15781

Stefoni, C. (2009). Migración, género y servicio doméstico. Mujeres peruanas en Chile. En M. E. Valenzuela \& C. Mora (Eds.), Trabajo doméstico: Un largo camino hacia el trabajo decente. Organizacion Internacional del Trabajo.

Tijoux, M. E. (2013). Niños(as) marcados por la inmigración peruana: Estigma, sufrimientos, resistencias. Convergencia, 20(61), 83-104. http://www.scielo.org.mx/scielo. php?script=sci_arttext\&pid=S1405-14352013000100004

Tijoux, M. E. (2014). El Otro inmigrante "negro" y el Nosotros chileno. Un lazo cotidiano pleno de significaciones". Boletín Onteaiken, 17, 1-15. http://onteaiken.com.ar/ver/ boletin17/art-tijoux.pdf

Turner, J. C., Brown, R. J., \& Tajfel, H. (1979). Social comparison and group interest in ingroup favouritism. European Journal of Social Psychology, 9(2), 187-204. https://online library.wiley.com/doi/pdf/10.1002/ejsp.2420090207

Turner, R., \& Feddes, A. (2011). How intergroup friendship works: A longitudinal study of friendship effects on outgroup attitudes. European Journal of Social Psychology, 41(7), 914-923. https://doi.org/10.1002/ejsp.843

Urzúa, A., Boudon-Torrealba, S., \& Caqueo-Urízar, A. (2017). Salud mental y estrategias de aculturación en 
inmigrantes colombianos y peruanos en el norte de Chile. Acta Colombiana de Psicología, 20(1), 70-79. https://doi. org/10.14718/ACP.2017.20.1.5

Urzúa, A., Caqueo-Urízar, A., Carvajal, C. C., \& Páez, L. R. (2017). ¿El apoyo social mediatiza el efecto negativo del estrés por aculturación en la salud? Un estudio en colombianos y peruanos inmigrantes en el norte de chile. Interciencia, 42(12), 818-822. https://www.interciencia.net/wp-content/ uploads/2017/12/818-CAQUEO-42-12.pdf

Urzúa, A., Delgado, E., Rojas, M., \& Caqueo, A. (2017). Social Well-Being Among Colombian and Peruvian Immigrants in Northern Chile. Journal of Immigrant and Minority Health, 19(5), 1140-1147. https://doi.org/10.1007/ s10903-016-0416-0

Urzúa, A., Ferrer, R., Godoy, N., Leppes, F., Trujillo, C., Osorio, C., \& Caqueo-Urízar, A. (2018). The mediating effect of self-esteem on the relationship between perceived discrimination and psychological well-being in immigrants. PLOS ONE, 13(6), e0198413. https://doi.org/10.1371/jour nal.pone.0198413

Valdes, C. (2018). The changing demographic landscape of Chile: Crime, and recent immigration patterns. Encrucijada Americana, 10(2), 47-54. https://dialnet.unirioja.es/descar ga/articulo/7056741.pdf

Van Sonderen, E., Sanderman, R., \& Coyne, J. C. (2013). Ineffectiveness of Reverse Wording of Questionnaire Items: Let's Learn from Cows in the Rain. PLOS ONE, 8(7). https://doi.org/10.1371/journal.pone.0068967
Vera-Villarroel, P., Silva, J., Celis-Atenas, K., \& Pavez, P. (2014). Evaluación del cuestionario SF-12: Verificación de la utilidad de la escala salud mental. Revista Médica de Chile, 142(10), 1275-1283. http://dx.doi.org/10.4067/ S0034-98872014001000007

Vicente, B. P., Kohn, R., Saldivia, S. B., \& Rioseco, P. S. (2007). Burden of psychiatric diseases in Chile. Revista Médica de Chile, 135(12), 1591-1599. https://doi.org// S0034-98872007001200014

Ware, J. E., Kosinski, M., \& Keller, S. D. (1996). A 12-Item ShortForm Health Survey: Construction of scales and preliminary tests of reliability and validity. Medical Care, 34(3), 220233. https://doi.org/10.1097/00005650-199603000-00003

Williams, D. R., \& Mohammed, S. A. (2009). Discrimination and racial disparities in health: Evidence and needed research. Journal of Behavioral Medicine, 32(1), 20-47. https://doi.org/10.1007/s10865-008-9185-0

Wold, B. (1995). HBSC: A World Health Organization CrossNational Survey. Resource Package of Questions 19931994. Univerity of Bergen.

Yañez, S., \& Cárdenas, M. (2010). Estrategias de aculturación, indicadores de salud mental y bienestar psicológico en un grupo de inmigrantes sudamericanos en Chile. Salud \& Sociedad, 1(1), 51-70. https://dialnet.unirioja.es/descarga/ articulo/4016251.pdf 